\title{
Evolution of rivulets during spreading of an impinging water jet on a rotating, precoated substrate ${ }^{\circ}$
}

Cite as: Phys. Fluids 31, 082104 (2019); https://doi.org/10.1063/1.5109806

Submitted: 13 May 2019 . Accepted: 03 July 2019 . Published Online: 07 August 2019

Andrew S. Ylitalo (D), Daniel J. Walls (D), David S. L. Mui, John M. Frostad (D), and Gerald G. Fuller (D)

\section{COLLECTIONS}

F This paper was selected as Featured

\section{CAPTURE WHAT'S POSSIBLE}

\section{WITH OUR NEW PUBLISHING ACADEMY RESOURCES}

Learn more $\Theta$
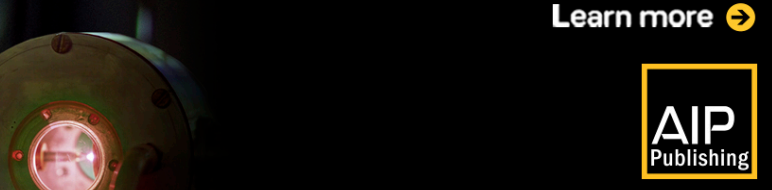


\title{
Evolution of rivulets during spreading of an impinging water jet on a rotating, precoated substrate $\mathrm{F}$ (Sc)
}

\author{
Cite as: Phys. Fluids 31, 082104 (2019); doi: 10.1063/1.5109806 \\ Submitted: 13 May 2019 Accepted: 3 July 2019 • \\ Published Online: 7 August 2019
}



Andrew S. Ylitalo, ${ }^{1,2}$ (D) Daniel J. Walls, ${ }^{1,3}$ (D) David S. L. Mui, ${ }^{4}$ John M. Frostad, ${ }^{1,3,5, a)}$ (D) and Gerald G. Fuller ${ }^{1, b)}$ (D)

\begin{abstract}
AFFILIATIONS
${ }^{1}$ Department of Chemical Engineering, Stanford University, Stanford, California 94305, USA

2Division of Chemistry and Chemical Engineering, California Institute of Technology, Pasadena, California 91125, USA

${ }^{3}$ Department of Chemical and Biological Engineering, University of British Columbia, Vancouver,

British Columbia V6T 1Z3, Canada

¿Lam Research Corporation, Fremont, California 94538, USA

${ }^{5}$ Food Science, University of British Columbia, Vancouver, British Columbia V6T 1Z3, Canada
\end{abstract}

a) Electronic mail: jfrostad@mail.ubc.ca

b) Electronic mail: ggf@stanford.edu

\begin{abstract}
The spreading of a liquid film across a rotating surface is inherently unstable due to the centrifugal force, which causes the formation of rivulets along the spreading front. This instability produces a rich diversity of spreading patterns and is important to control for the optimization of spin-coating and spin-rinsing of silicon wafers during the fabrication of microelectronics. The present work is an experimental investigation of the evolution of rivulets arising from this instability during the spreading of an impinging water jet across a rotating substrate that is precoated with a thin, aqueous film. To characterize these rivulets, we developed a high-speed imaging apparatus and image-processing software that traces the spreading front over time. We show how the morphology of the spreading front is qualitatively affected by varying the Reynolds number of the impinging jet, the ratio of centrifugal to Coriolis forces, and the type of liquid used to precoat the substrate. For quantitative analysis of rivulets, we measured the "compactness ratio" of the spreading front, which quantifies deviation from a circular spreading front. We used the compactness ratio to demonstrate that rivulets are suppressed most strongly at low rotation rates, at high flow rates, and on substrates precoated with water, although with notable exceptions.
\end{abstract}

Published under license by AIP Publishing. https://doi.org/10.1063/1.5109806

\section{INTRODUCTION}

The spreading of a liquid across a solid substrate is a classic problem in fluid dynamics. ${ }^{1,2}$ When liquids spread as a result of shear stresses ${ }^{3,4}$ or a body force, thin liquid streams, known as "rivulets" or "fingers" (terms typically used interchangeably), can emerge from the spreading front. In the case of spreading due to a body force, the rivulets have been shown to emerge as a result of an instability in the capillary ridge along the spreading front. Thicker regions along the capillary ridge experience weaker viscous resistance than thinner regions, which results in a greater net force in the direction of spreading and causes them to protrude beyond the thinner regions. The liquid then preferentially flows through these protrusions, elongating them to form rivulets, which may further break up into smaller rivulets. ${ }^{6}$ Most studies of rivulets that form in this way have investigated spreading down an inclined surface due to gravity ${ }^{7-11}$ or spreading across a rotating substrate due to the centrifugal force. ${ }^{12-14}$ This paper expands on the latter case by considering the effects of supplying liquid with an impinging jet and precoating the substrate with a thin liquid film.

In addition to academic interest, study of the rivulets that form due to the centrifugal force have a primary application in the improvement of industrial spin coating (spreading of a fixed volume of liquid) and spin rinsing (spreading of a continuously supplied liquid), such as are commonly used during the fabrication 
of microelectronics on silicon wafers. While spin coating has a long history of scientific investigation, interest in spin rinsing emerged relatively recently. This interest in spin rinsing has been in part motivated by its potential to become an environmentally friendly alternative to the industry standard for cleaning silicon wafers during fabrication, the "RCA clean" process, which employs large quantities of harsh chemicals. One such alternative, invented by Mui et al., proposes to clean a silicon wafer by first spin coating the wafer with a polymer solution designed to collect contaminants from printed microstructures and then spin rinsing the wafer to remove the polymer solution along with the collected contaminants. ${ }^{15}$ One of its primary drawbacks, however, is the formation of rivulets, which can accumulate contaminants along their stagnant edges. Additionally, the formation of rivulets results in nonuniform spreading that increases the time and liquid required to fully rinse the substrate, a problem shared by spin rinsing and spin coating alike. Methods have been proposed to prevent the formation of rivulets on rotating substrates using electric ${ }^{16}$ and magnetic ${ }^{17}$ fields, but these techniques require added particulates, which are not suitable for cleaning applications. Therefore, studies like the present work that examine the formation and evolution of rivulets on rotating horizontal substrates are essential for the development of effective spin coating and spin rinsing, particularly of silicon wafers.

Melo et al. performed the first thorough experimental study on the formation of rivulets from a liquid droplet spreading over a rotating, horizontal substrate. ${ }^{12}$ They observed that a droplet of viscous liquid forms a ridge along the outer perimeter, which eventually adopts a polygonal morphology. At this point, rivulets pinch off radially from the vertices. They found that the radius of the droplet, thickness of the droplet, number of rivulets, and critical radius at which rivulets first formed could be described by similarity curves. Others extended the work of Melo et al. by analyzing the Fourier power spectrum of the spreading front to identify the unstable modes and the critical radius. ${ }^{13,14,18}$ Studies of the spreading of droplets with much lower viscosity observed that the rivulets spiral due to the stronger Coriolis force. ${ }^{19-21}$ These rivulets are also more irregular than those observed during the spreading of viscous droplets, with some rivulets emerging from other rivulets. ${ }^{19}$

Researchers have also investigated the formation of rivulets during the spreading of liquid supplied by a continuous source, generally a vertical impinging jet. Togashi et al. showed that during the spreading of aqueous glycerol solutions supplied by an impinging jet, the critical radius at which rivulets emerged increased roughly as the flow rate to the one-third power. ${ }^{22}$ Others considered the case of an impinging jet that dispensed a fixed volume of liquid at a constant rate. They found that spreading is initially dominated by the impinging jet, but later resembles droplet spreading. ${ }^{23,24}$

Additionally, researchers have investigated the effects of altering the surface of the substrate. Some have considered the effects of altering the geometry of the substrate, ${ }^{11,25}$ although the present paper focuses on flat substrates. Others have precoated the substrate with a thin film of liquid before spreading. For example, Melo et al. noted that rivulets that form during the spreading of a droplet of silicone oil on a rotating disk are suppressed when the disk is precoated with a sufficiently thick film of silicone oil. ${ }^{12}$ Veretennikov et al. made similar observations for the spreading of droplets of silicone oil, of castor oil, and of glycerol on both inclined planes and rotating substrates, ${ }^{26}$ as did de Bruijne and Lammers for the spreading of propanol on a rotating substrate. ${ }^{19}$ More recently, Sahoo et al. showed that spreading of a liquid drop over a larger liquid drop on a rotating substrate exhibits faster spreading and a larger number of rivulets than the spreading of a single liquid drop over a rotating substrate, ${ }^{27}$ which has been modeled under axisymmetric conditions by Sahoo et al. ${ }^{28}$

Most previous studies of rivulets on rotating substrates have focused on their initial formation. Those that have studied the evolution of their morphology after formation have focused on the spreading of viscous liquids on bare substrates, which produce regular, radial rivulets. The present work explores the rich diversity of morphologies of the spreading front that arise during spreading from an impinging jet of water over a rotating substrate precoated with a thin, aqueous film. To overcome the challenge of identifying the spreading front during spreading on precoated substrates, we developed an experimental apparatus that tracks the spreading front with a greater sensitivity than previously reported. Our apparatus allows us to quantitatively study how (1) the rotation rate of the substrate, (2) the flow rate of the impinging jet, and (3) the type of liquid film precoating the substrate affect both the morphology of the spreading front and its evolution over time. We refrain, however, from discussing the rate of spreading of the spreading front, which is investigated in a companion paper by Walls et al. ${ }^{29}$

First, in Sec. II, we describe an experimental apparatus for performing and visualizing spin rinsing, as well as the imageprocessing software developed by the authors to track the spreading front during these experiments. We present images of these experiments along with their traced spreading fronts in Sec. III A, which we use to demonstrate qualitative trends in the morphology of the spreading front under different experimental conditions. Next, Sec. III B introduces the use of the compactness ratio to quantitatively measure the degree of perturbation of the spreading front. We use the compactness ratio to demonstrate time-dependent trends in rivulet morphology and discuss more rigorously the effects of experimental conditions on the formation and growth of rivulets. Finally, Sec. IV summarizes the major findings of this paper.

\section{EXPERIMENTAL METHODS}

\section{A. Apparatus}

Experiments were performed on a custom-built spin-rinsing apparatus (Fig. 1). In this apparatus, the motor of a spin-coater from Lam Research is used to spin a circular substrate at a constant speed. The motor was controlled via TCP/IP connection by a Yaskawa electronic drive, programmed using the accompanying MotionWorks IEC software, which permitted rotation rates from 0 to $1000 \mathrm{rpm}$. In all experiments, the substrate was rotated counterclockwise as viewed from above. Above the motor, the substrate is held in the wafer chuck by six spring-loaded pins, which are equally spaced along its circumference. These pins grip the edge of the substrate, suspending it approximately $1 \mathrm{~cm}$ above the surface of the chuck. 


\section{1}

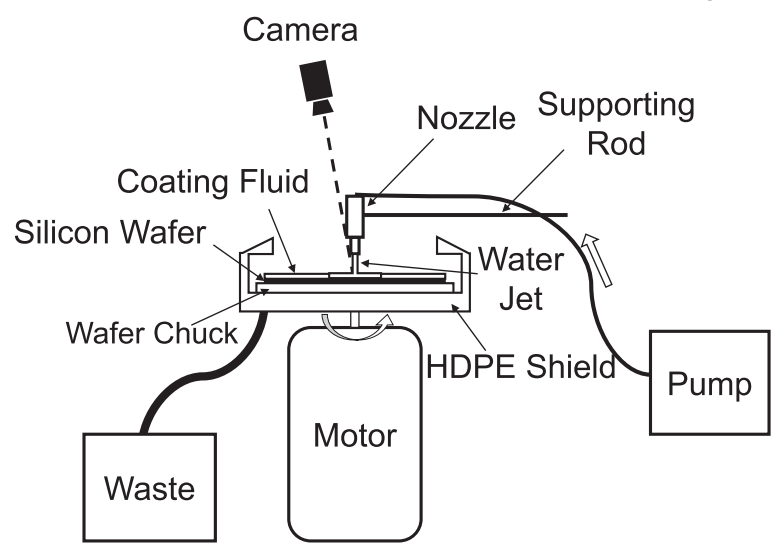

FIG. 1. Diagram of the spin-rinsing apparatus consisting of an industrial-grade motor and wafer chuck designed for $300 \mathrm{~mm}$ silicon wafers, an HDPE shield for waste collection, a positive-displacement pump, semirigid tubing, a vertically oriented nozzle centered along the axis of rotation, and a high-speed camera positioned off-center to be able to see the impact of the impinging jet. The silicon wafer reflects a patterned background designed to enhance contrast between the liquid film coating and rinsing flow.

A high-density polyethylene (HDPE) shield around the substrate and wafer chuck collected liquid runoff during experiments into a drain connected to a waste container.

The substrates were hydrophilic, unpatterned 300-mmdiameter silicon wafers. As a result of the hydrophilicity of the substrates, aqueous solutions would form contact angles close to zero. The substrates were obtained from Lam Research and kept in an F300 AutoPod ${ }^{\circledR}$ Forward Opening Unified Pod (FOUP) wafer carrier to prevent contamination before initial use. Between experiments, the substrates were sealed in individual plastic containers.

The impinging jet of water was emitted from a nozzle with an internal diameter of $1 / 4$ in. $(6.35 \mathrm{~mm})$. The nozzle was oriented vertically along the axis of rotation of the substrate and was elevated $4.0 \mathrm{~cm}$ above the substrate. To produce the impinging water jet, deionized water was pumped from a tank below by a Cole-Parmer positive displacement digital gear pump with a magnetic drive capable of providing flow rates of $50-3000 \mathrm{ml} / \mathrm{min}$. At the start of a spin-rinsing experiment, a solenoid valve switched the destination of the flow from the waste container to the jet nozzle while the pump was running. This method ensured that the impinging jet would immediately flow at the desired flow rate, as in Hsu et al. ${ }^{30}$ and Walker et al. ${ }^{3}$

Rinsing flow was video-recorded from above the substrate using a $25 \mathrm{~mm}$, F1.4 lens from Edmund Optics on a $4 \mathrm{~GB}$ Model $120 \mathrm{~K}$ Photron FASTCAM SA3 high-speed camera. The aperture of the lens was adjusted to increase the depth of focus such that the rinsing flow on the substrate and the reflection of the patterned background above the substrate were simultaneously in focus. The camera was positioned off-center and at an angle to have a direct line of sight to the center of the substrate. Illumination of the substrate was provided by four $250 \mathrm{~W}$ halogen work lamps equally spaced around the HDPE shield and directed toward the patterned background. To improve image processing, we dampened the flickering in the lamps from the AC wall current $(120 \mathrm{~V}, 60 \mathrm{~Hz})$ using a low-cost, custom-built rectifier circuit. The rectifier circuit consisted of a $3300 \mu \mathrm{F}$ aluminum electrolytic capacitor (EPCOS/TDK, \#B43456-A9338-M), a rectifier bridge (Diodes Incorporated, \#GBJ-2004-F), and a thermistor (Ametherm, \#SL32 5R020).

Because the precoated films were aqueous, distinguishing them from the rinsing flow of the water jet was challenging in experiments where the substrate was precoated. To enhance the contrast between the rinsing flow and the precoated film, a patterned background with a smoothly varying gradient between black and white was placed in the reflection of the substrate (see Fig. 2). While the smooth surface of the precoated film reflected the true background pattern, the ripples on the surface of the rinsing flow distorted the pattern. We tracked these distortions by thresholding the difference between the given image and a reference image taken before rinsing flow began. Finally, we identified the rinsing flow in a given image by compiling the distortions from the previous frames.

A simpler "chessboard" pattern of alternating white and black squares was also considered, but was not used due to two disadvantages. First, slight deflections of the substrate from the impinging jet distorted the reflected background pattern enough that the sharp gradient between squares produced artifacts when subtracting the background image. Second, the ripples in the rinsing liquid were sometimes too small to distort the background pattern enough to reflect a different shade from the coating liquid. The smoothly varying background pattern resolved both of these issues.

\section{B. Materials}

Deionized water was used for all aqueous rinsing and coating solutions. Some experiments used substrates coated with aqueous

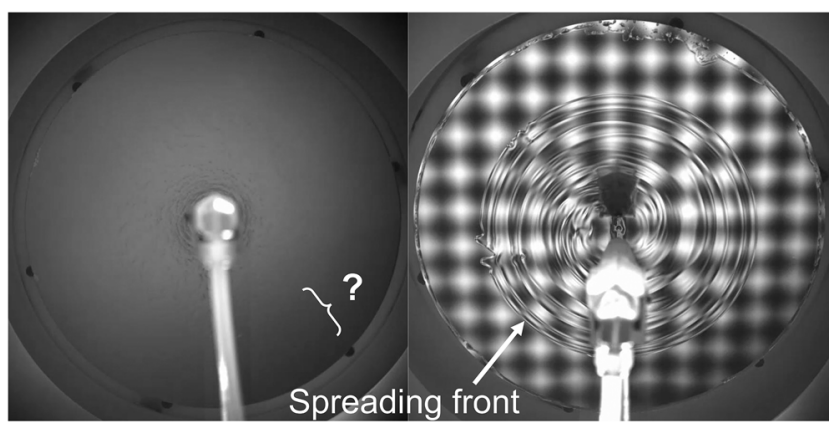

FIG. 2. Overhead views of a rotating $(250 \mathrm{rpm})$ silicon wafer coated with a thin film (approximately $15 \mu \mathrm{m}$ ) of water as water from an impinging jet $(390 \mathrm{ml} / \mathrm{min})$ spread radially across it. In both images, the spreading front reached approximately the same radius. Left. The background above the silicon wafer is uniformly colored. The spreading front is estimated to be somewhere within the bracket, but cannot be distinguished from the liquid film coating. Right. The silicon wafer reflects a patterned background with a smoothly varying gradient between black and white repeated in a grid pattern. The spreading front and other minor ripples in the surface of the liquid can be clearly distinguished. 
solutions of sodium dodecyl sulfate (SDS, Invitrogen Life Technologies Lot \#1158817), which was used without further purification. SDS was selected as the surfactant because it is an anionic surfactant, which we expected not to be attracted to the negatively charged silicon wafer used as the substrate. Isotherms for SDS in water providing surface tension as a function of concentration can be found in Fig. 4 of Frostad et al. ${ }^{32}$ Aqueous solutions of SDS at $3.0 \mathrm{mM}, 6.5 \mathrm{mM}$ (the critical micelle concentration or "CMC"), and $10 \mathrm{mM}$ were used. Although the $6.5 \mathrm{mM}$ and $10 \mathrm{mM}$ solutions had the same surface tension, comparing them allowed us to test for effects of dilution of the surfactant that may have resulted from mixing of the rinsing and coating liquids during spreading. Nevertheless, because we observed that the spreading front was qualitatively similar for all three concentrations of SDS, we only present results for spreading over substrates coated with $3.0 \mathrm{mM}$ SDS solutions.

To clean the substrate (procedure described below), we used the cleaning solution from the "Standard Clean-1" step of the standard RCA clean process. This solution comprises a 5:1:1 ratio of deionized water, $38 \%$ aqueous ammonium hydroxide $\left(\mathrm{NH}_{4} \mathrm{OH}\right)$ solution, and $30 \%$ aqueous hydrogen peroxide $\left(\mathrm{H}_{2} \mathrm{O}_{2}\right)$ solution and was contained in a PTFE basin.

\section{Procedure}

\section{Cleaning substrates}

Within $48 \mathrm{~h}$ before experiments, substrates were submerged in a Standard Clean-1 (SC-1) solution (see Sec. II B for composition) at $50^{\circ} \mathrm{C}$ for $36 \mathrm{~min}$ in a cleanroom. The substrate was subsequently rinsed with deionized water and dried with an air jet before being sealed in a plastic container for storage until removal for experiments.

\section{Spin coating substrates}

In some experiments, the substrate was precoated with a thin liquid film of either deionized water or an aqueous solution of SDS before rinsing with the impinging water jet. To coat the substrate, $30 \mathrm{ml}$ of the desired coating solution was first dispensed with an electronic pipette gun at the center of the substrate. The substrate was then spun according to a spinning routine that was determined by integrating Emslie's differential equation for the change in thickness of a Newtonian liquid film on a rotating disk with respect to time, accounting for time-varying rotation rates during start-up. The parameters of the spinning routine were selected such that the predicted thickness of the film of coating solution at the moment the water jet emerged from the jet nozzle was approximately $15 \mu \mathrm{m}$. If the coating liquid film produced by this procedure had dry spots, the experiment was not considered during the analysis. Spin coating the substrate at $400 \mathrm{rpm}$ was found to result in the fewest dry spots.

\section{Spin rinsing substrates}

The impinging jet was immediately initiated at the desired flow rate by switching the flow from the waste stream to the impinging jet using a solenoid valve. As a result, the impinging jet often produced a splash upon impact on the substrate, even causing the substrate to deflect slightly downward in some cases. Rinsing flow continued until the substrate was completely coated by the rinsing liquid.

We also considered gradually increasing the flow rate of the jet to the desired flow rate because the jet did not splash or deflect the substrate upon impact in this case. We used the solenoid valve, however, because it provided more consistent flow among different experiments.

\section{Drying substrates}

In between experiments, the rinsing liquid remaining on the substrate was dried with a jet of compressed air directed radially outward while spinning the substrate at $300 \mathrm{rpm}$ for approximately $2 \mathrm{~min}$.

\section{Image processing}

The spreading front was identified and analyzed using imageprocessing software developed by the authors for this work. Our method differs from previous methods by distinguishing the rinsing liquid based on distortion of the background pattern by ripples along the liquid surface, which does not require a parallel overhead light source ${ }^{12}$ or dying of the fluid ${ }^{13,27,34}$ for visualization.

In our method, we first removed distortion of the image due to the oblique angle of the camera using a homographic transformation that restored the circular shape of the substrate. Next, a reference image of the substrate before rinsing was subtracted from a given image of the rinsing flow. The result was thresholded to identify the rinsed region based on distortion of the background pattern by ripples along the surface of the rinsing flow. The accuracy was improved by keeping track of which areas had already been marked as "rinsed" in the previous frame so that only newly rinsed regions needed to be identified in each frame. In some cases, droplets splashed beyond the spreading front, and these were excluded from the rinsed region. Because some of the spreading front was obstructed by the steel supporting bar that held the nozzle for the impinging jet, the image-processing software stored the spreading front from previous frames to approximate the obstructed segment of the spreading front. This approximation introduced some error in the measurement of the perimeter of the spreading, but we found that the error was negligible in comparison to variations in the perimeter among different repetitions of the same experiment. Due to the sharp features of the reflected image of the nozzle near the center of the substrate, the image-processing software could not identify the full spreading front until it reached a radius of about $4 \mathrm{~cm}$. Consequently, we do not present data for spreading below a radius of $4 \mathrm{~cm}$ in this paper. In some cases, rivulets swept across regions of the substrate and left behind a liquid film that was much thinner than the rest of the spreading film. Visually, these regions of the substrate were often difficult to distinguish from regions over which no liquid had flowed. The imageprocessing software, however, "remembers" that these regions have been rinsed and includes them in the rinsed region. Additionally, to reduce the influence of the substrate's edge, we masked pixels in the image beyond a radius of $14 \mathrm{~cm}$ (approximately $95 \%$ of the radius of the substrate). Finally, the perimeter of the rinsed region was traced to identify the spreading front. Every fifth point was used in calculations to smooth out the effects of pixelation along the boundary. 


\section{RESULTS AND DISCUSSION}

\section{A. Morphology of the spreading front}

\section{Spreading front over time}

Within a single experiment, we observed that the spreading front progressed through several distinct morphologies over time. Such a progression is shown in Fig. 3. In this experiment, water from the impinging jet was flowing at $1290 \mathrm{ml} / \mathrm{min}$ over a bare substrate that was rotating at $120 \mathrm{rpm}$. The spreading front initially had a circular shape. As the spreading front was perturbed, it formed short, wide lobes, which lasted briefly until they grew to be long, narrow rivulets. For most cases, we observed that rivulets became longer as compared to their width over time, resulting in similar progressions toward long, narrow rivulets. One notable exception is the case of water spreading over a water-coated substrate. In this case, the elongation of rivulets was noticeably suppressed after their initial formation (see Sec. III B 4 for further discussion).

\section{Classifying the morphology of the spreading front}

Given the time dependency and variation of the morphology of the spreading front observed throughout the course of a single experiment, for simplicity, we focus this section of our analysis on the morphologies of spreading fronts observed when the spreading front neared the edge of the substrate. This approach provides a more consistent comparison among experiments with different rotation rates of the substrate, flow rates of the impinging jet, and coating films on the substrate. In particular, we examined spreading fronts whose maximal extent reached $90 \%$ of the radius of the substrate $(13.5 \mathrm{~cm})$. This radius was selected to show the spreading front at as large a radius as possible before edge effects along the outer edge of the substrate disturbed the spreading. We visually classified these morphologies into seven categories based on the rivulets' aspect ratios, degree of spiraling, and degree of regularity. As such, the following qualitative analysis is not meant to be a rigorous definition, but rather a descriptive grouping of rivulet morphologies.

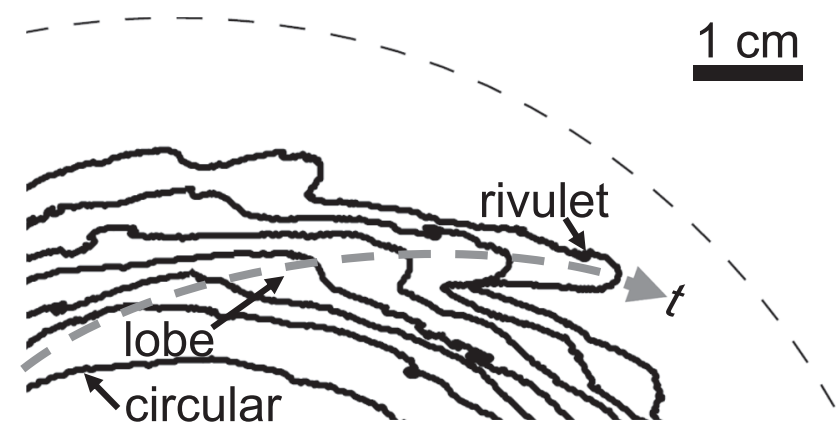

FIG. 3. Outlines (black lines) of the spreading front of water from an impinging jet with a flow rate of $1290 \mathrm{ml} / \mathrm{min}$ spreading across a bare substrate rotating at $120 \mathrm{rpm}$, as seen from the reference frame of the substrate. Frames are taken every $60 \mathrm{~ms}$, except the first frame, which is taken $150 \mathrm{~ms}$ before the second for clarity. The dashed arrow indicates the growth of a lobe into a rivulet from an initially circular spreading front. The dashed arc around the spreading front marks the edge of the viewing window (at a radius of approximately $14 \mathrm{~cm}$ ).
In making these classifications, we distinguish protrusions from the spreading front that are "radial," "spiraled," and "straight." "Radial" protrusions extend radially without a noticeable azimuthal component. "Spiraled" protrusions have a noticeable curvature in the azimuthal direction. "Straight" protrusions do not have a noticeable curvature when considering their extent from base to tip, but are not necessarily directed in a purely radial direction.

Figure 4 shows representative examples of each category of spreading front morphology and the conditions under which they were observed. Figures 4(a) and 4(b) show the categories of the spreading front as a function of the rotation rate of the substrate and the flow rate of the impinging jet. The categories for both bare substrates and substrates coated with $3 \mathrm{mM}$ SDS are presented in Fig. 4(a) because of the similarity in their morphologies. Figure 4(b) shows the categories for substrates coated with water. Figure 4(c) (Multimedia view) shows the spreading front of a representative example from each of the seven categories with the contour traced by image processing. Figure 4(d) shows a side-by-side comparison of the contours of the spreading fronts shown in Fig. 4(c) (Multimedia view).

The seven categories of spreading front morphology that we recorded are described as follows. Clearly the distinctions between some of these categories are not sharp, but we believe that key distinctions can be made that warrant this categorization.

1. Circular: the spreading front is roughly circular with no protrusions. See Fig. 4(c(1)) (Multimedia view).

2. Lobed: the spreading front has protrusions with an aspect ratio (base-to-tip distance to azimuthal extent) much less than 1. See Fig. 4(c(2)) (Multimedia view).

3. Radial teeth: the spreading front has radial protrusions with an aspect ratio around 1. See Fig. 4(c(3)) (Multimedia view).

4. Spiraled teeth: the spreading front has spiralled protrusions with an aspect ratio around 1. See Fig. 4(c(4)) (Multimedia view).

5. Smooth rivulets: the spreading front has spiraled protrusions with an aspect ratio between 1 and 10. See Fig. 4(c(5)) (Multimedia view).

6. Spindly rivulets: the spreading front has straight protrusions with an aspect ratio greater than 10 , typically having a wider, triangular base. See Fig. 4(c(6)) (Multimedia view).

7. Turbulent: thin protrusions with no regularity in length, width, or orientation emerge from a highly asymmetric spreading front. See Fig. 4(c(7)) (Multimedia view).

First, we will discuss bare substrates and substrates coated with $3 \mathrm{mM}$ SDS [Fig. 4(a)]. When the substrate was stationary, the flow spread smoothly with a circular front (1). When the substrate rotated at $30 \mathrm{rpm}$, the spreading front became slightly perturbed by the centrifugal force, leading to the protrusion of wide, smooth lobes out from the spreading front (2). As the rotation rate was increased, between $120 \mathrm{rpm}$ and $250 \mathrm{rpm}$, the initial perturbations along the spreading front grew into smooth, thin rivulets (5). These rivulets were observed to curve and spiral as a result of the increasing strength of the Coriolis force relative to the centrifugal force, for which we define a Rossby number

$$
R o \equiv \frac{f_{\text {cent }}}{f_{\text {Cor }}}=\frac{\Omega r}{2 v},
$$




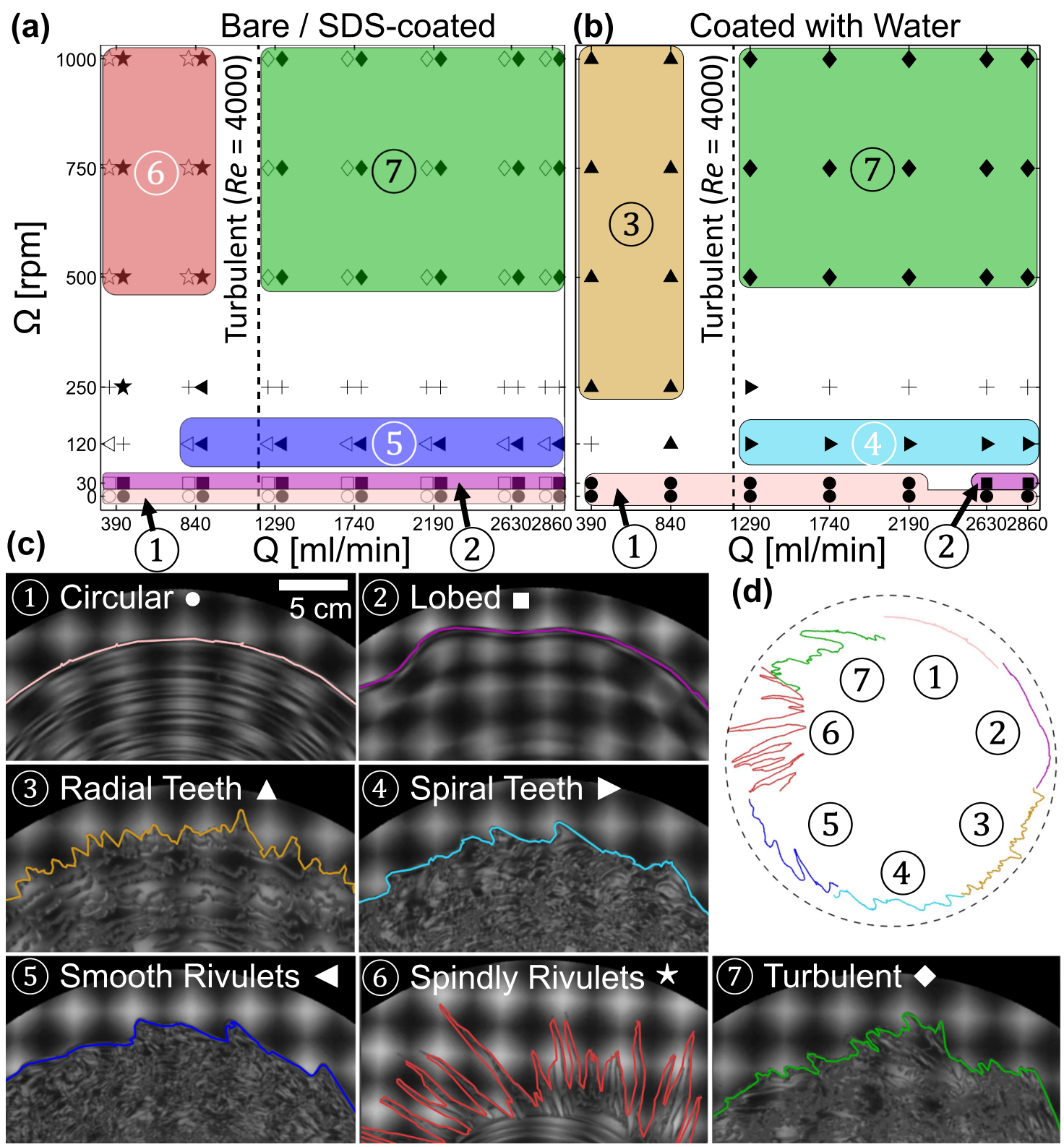

FIG. 4. The seven categories of spreading front morphologies observed when the spreading front neared the edge of the substrate. (a) Plot of the morphology of the spreading front observed for both bare substrates (open symbols) and substrates coated with a film of $3 \mathrm{mM}$ SDS (closed symbols) at different flow rates and rotation rates. See the legend below. Clusters of experiments that fell in the same category are shaded to guide the eye. (b) Corresponding plot to (a) for substrates coated with water. (c) Zoomed-in images of spin-rinsing experiments representative of each category with the spreading front traced by image processing: (1) $\bullet-$ circular, (2) -lobed, (3) $\Delta-$ radial teeth, (4) - -spiral teeth, (5) $\-$ smooth rivulets, (6) $\star-$ spindly rivulets, and (7) - -turbulent rivulets. The plus sign + indicates a transition in morphology between the immediately adjacent symbols above and below. (d) Segments of spreading fronts from experiments performed under the same conditions as those shown in (c) arranged on the same image for comparison. Multimedia views: (c): (1) https://doi.org/10.1063/1.5109806.1; (2) https://doi.org/10.1063/1.5109806.2; (3) https://doi.org/10.1063/1.5109806.3; (4) https://doi.org/10.1063/1.5109806.4; (5) https://doi.org/10.1063/1.5109806.5; (6) https://doi.org/10.1063/1.5109806.6; (7) https://doi.org/10.1063/1.5109806.7

where $\Omega$ is the rotation rate, $r$ is the radial position, and $v$ is the radial velocity.

To estimate $R o$, we use the results from Walls et al., ${ }^{29}$ which demonstrated that under these conditions (Region 2 in Walls et al.), the radius of the spreading front after the initial splash is exponential in time with a time constant equal to the inverse of the rotation rate, $r(t)=A e^{\Omega t}$, for some positive real constant $A$. Taking a derivative with respect to time, $v(t)=A \Omega e^{\Omega t}$. Therefore, based on Eq. (1), 
$R o=1 / 2$ after the initial splash and remains constant throughout spreading. Because $R o<1$, the Coriolis force is stronger than the centrifugal force in this case. A stronger Coriolis force than the centrifugal force means that rivulets should curve azimuthally more as they progress outward radially, in agreement with our observations (see Fig. 3, for example).

At higher rotation rates $(500-1000 \mathrm{rpm})$ and low flow rates $(390 \mathrm{ml} / \mathrm{min}$ and $840 \mathrm{ml} / \mathrm{min}$ ), rivulets quickly elongated beyond the rest of the spreading front shortly after first emerging. The resulting rivulets were evenly spaced and had broader, triangular bases, which narrowed to thin, straight, "spindly" streams (6). When they first formed, however, they spiralled like smooth rivulets (5), as was previously observed for a spreading droplet under similar conditions. ${ }^{2}$ Results from Walls et al. can be used to show that the ratio of centrifugal force to Coriolis force increases during this transition from spiral to straight rivulets. Under these conditions, the spreading rate follows a power-law (Region 3 in Walls et al.), $r(t)=A\left(t-t_{0}\right)^{3 / 2}$, so $v(t)=\frac{3}{2} A\left(t-t_{0}\right)^{1 / 2}$. Therefore, based on Eq. (1), Ro $=\Omega\left(t-t_{0}\right) / 3$, which increases over time, unlike the case of smooth rivulets (5). An example of this transition is shown in Fig. 5, in which the ratio of the centrifugal force to the Coriolis force Ro increases from roughly $1 / 2$ to 1 over the course of the experiment. We note that on larger substrates, we would expect the rivulets to become increasingly radial.

At higher flow rates $(1290-2860 \mathrm{ml} / \mathrm{min})$, rivulets had a more irregular, transient shape, and the bulk of the spreading liquid was highly asymmetric. At higher rotation rates (500-1000 rpm), the rivulets were thinner and were quickly engulfed by the spreading front after forming. We broadly categorized these morphologies as "turbulent" (7) because the Reynolds number of the impinging jet is in the turbulent regime. The Reynolds number of the impinging jet was defined as

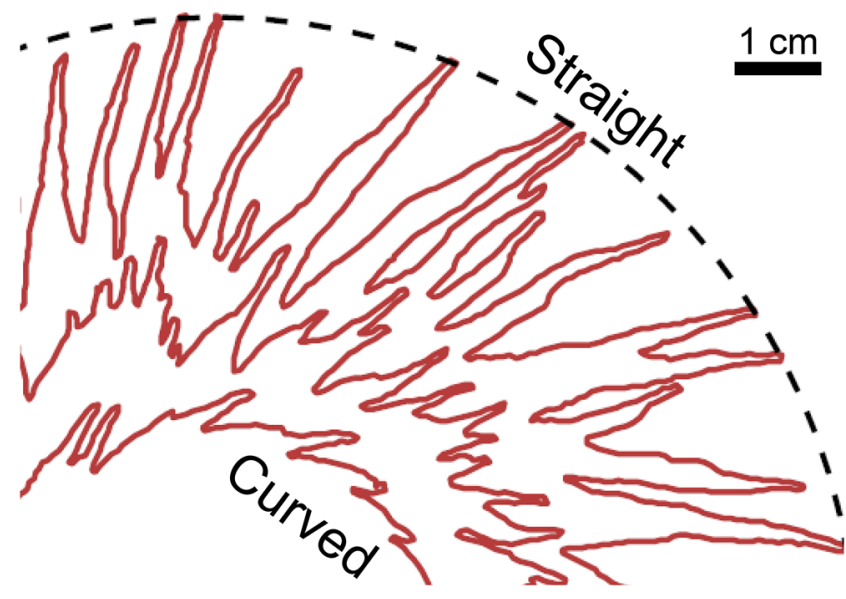

FIG. 5. Outlines (red) of the spreading front on a bare substrate rotating at $750 \mathrm{rpm}$ and supplied by an impinging jet at $390 \mathrm{ml} / \mathrm{min}$, as seen from the reference frame of the substrate. Contours are taken at a mean radius of $5 \mathrm{~cm}$, $8 \mathrm{~cm}$, and $12 \mathrm{~cm}$. The circular dashed boundary marks the edge of the viewing window, with a radius of approximately $14 \mathrm{~cm}$. The Rossby number $R o=f_{\text {cent }} / f_{\text {cor }}$ increases from roughly $1 / 2$ to 1 as the mean radius increases from $5 \mathrm{~cm}$ to $12 \mathrm{~cm}$. The rivulets concurrently become straighter.

$$
R e=\frac{4 Q}{\pi d v},
$$

where $Q$ is the flow rate of the jet, $d$ is the diameter of the nozzle $(6.35 \mathrm{~mm})$, and $v$ is the kinematic viscosity of the rinsing liquid (water, $v=10^{-6} \mathrm{~m}^{2} / \mathrm{s}$ ). For flow rates at $1290 \mathrm{ml} / \mathrm{min}$ and above, $R e>4000$, the lower bound for turbulent flow. For comparison, at $390 \mathrm{ml} / \mathrm{min} R e \approx 1300$, which is below the upper bound for laminar flow through a pipe $(R e \approx 2100)$, and at $840 \mathrm{ml} / \mathrm{min} R e \approx 2800$, which is within the transitional flow regime. Consequently, we are not surprised that the morphology of the rivulets transitioned from spindly (6) to turbulent (7) as the impinging jet transitioned from laminar to turbulent.

Next, we will discuss substrates coated with water [Fig. 4(b)]. The spreading front was generally circular (1) for spreading on stationary substrates and substrates rotating at $30 \mathrm{rpm}$. At $30 \mathrm{rpm}$ and higher flow rates (above $2630 \mathrm{ml} / \mathrm{min}$ ), however, the spreading front became lobed. Previously, we observed that at $30 \mathrm{rpm}$, the spreading front became lobed on both bare and SDS-coated substrates for all flow rates. This difference suggests that the higher resistance along the spreading front on a bare or SDS-coated substrate may destabilize the spreading front.

At higher rotation rates (above $120 \mathrm{rpm}$ ), many stubby, triangular protrusions emerged from the spreading front, which we refer to as "teeth." These teeth typically formed at smaller radii than lobes formed (2), but did not grow much longer, remaining at an aspect ratio of around one. At nonturbulent flow rates $(390-840 \mathrm{ml} / \mathrm{min})$, the teeth predominantly pointed in the radial direction (3). In addition to occurring at similar rotation rates and flow rates as spindly rivulets (6), these radial teeth resembled their triangular bases (discussed above), although the teeth were smaller in both length and width than the triangular bases. For higher rotation rates $\Omega$ at nonturbulent flow rates, a larger number of thinner radial teeth formed along the spreading front, but they still maintained an aspect ratio of about one. At turbulent flow rates (above $1290 \mathrm{ml} / \mathrm{min}$ ) and intermediate rotation rates (120 rpm and $250 \mathrm{rpm}$ ), the teeth had a noticeable component in the azimuthal direction. We therefore categorized them as "spiralled teeth" (4). In addition to occurring at similar rotation rates and flow rates, spiralled teeth resembled smooth rivulets (5), except that they did not elongate nearly as much, remaining at an aspect ratio of around one. At turbulent flow rates (above $1290 \mathrm{ml} / \mathrm{min}$ ) and high rotation rates (500-1000 rpm), rivulets were similar to the turbulent rivulets (7) observed on bare substrates and substrates coated with SDS. This similarity in morphology for different coating liquids further supports our finding that at higher flow rates and rotation rates, the coating liquid has less influence on the rivulets.

\section{B. Compactness ratio}

\section{Definition}

To analyze rivulets quantitatively, we introduce a variation of the "compactness ratio," which is used in computer vision ${ }^{35}$ and zoning. ${ }^{36}$ Previous studies have used the Fourier power spectrum to describe the morphology of rivulets. ${ }^{13,14,18}$ While the Fourier power spectrum is more comprehensive, the compactness ratio provides a single-number summary of both amplitude and frequency of the undulations of the spreading front, which allows for a 
straightforward comparison between spreading fronts observed at different times or under different conditions.

In this paper, we define the compactness ratio as

$$
C=\frac{L}{2 \pi\langle R\rangle},
$$

where $L$ is the perimeter of the spreading front and $\langle R\rangle$ is the mean radius of the spreading front. The compactness ratio can thus be thought of as the ratio of the perimeter of the spreading front to the circumference of a circle with the same mean radius. Consequently, the compactness ratio of a smooth, closed contour is at least 1 , with higher values indicating a less compact spreading front. The use of the compactness ratio to describe rivulets has not been explored previously in the literature to our knowledge.

\section{Uncertainty in the measurement of the compactness ratio}

To estimate the uncertainty in our measurements of the compactness ratio, we performed five repetitions of a subset of experiments that spanned the full range of flow rate, rotation rate, and surface condition, and calculated the standard deviation. We found that at the lowest flow rate $(390 \mathrm{ml} / \mathrm{min})$, the standard deviation in the compactness ratio was below 0.2 for rotation rates below $500 \mathrm{rpm}$ and was below 0.3 for rotation rates $500 \mathrm{rpm}$ and higher. At the middle rotation rate $(250 \mathrm{rpm})$, the standard deviation was below 0.15 for flow rates below $2500 \mathrm{ml} / \mathrm{min}$ and was below 0.2 for flow rates above $2500 \mathrm{ml} / \mathrm{min}$. Some potential sources of systematic error that might have contributed to this uncertainty include the slight deflection of the substrate upon jet impact, evaporation of the coating liquid during spin coating, pixelation of the contours in the image, and obstruction of a portion of the substrate by the supporting rod for the jet. Nevertheless, the standard deviations observed among repetitions of experiments performed under the same conditions are estimated to be the largest source of error. We estimate that this uncertainty is less than $15 \%$ of the measured values of the compactness ratio, which suggests that the trends discussed below are robust.

\section{Connection to rivulet elongation}

To demonstrate the connection between rivulets and the compactness ratio, we plot the compactness ratio vs the mean radius of the spreading front in Fig. 6. At key points, we show the contour of the spreading front traced by our image-processing software for comparison. Initially, the spreading front appears roughly circular, corresponding to a compactness ratio of 1 . At a certain radius $(9 \mathrm{~cm}$, in this case), rivulets begin to form, and the compactness ratio concurrently begins a sudden and steady increase. The suddenness of the formation of rivulets-a characteristic of flow instability-is thus clearly captured by the compactness ratio. We note that the mean radius at which the compactness ratio begins this sudden increase roughly corresponds to the critical radius reported in the literature.

As the rivulets elongate, the compactness ratio increases. When the rivulets first reach the edge of the viewing window, the compactness ratio reaches its peak value. At this point, the compactness ratio steadily decreases to 1 as the gaps between the rivulets are filled in, resulting in a circular rinsed region once again. This behavior is seen in most experiments, although other observed trends are discussed
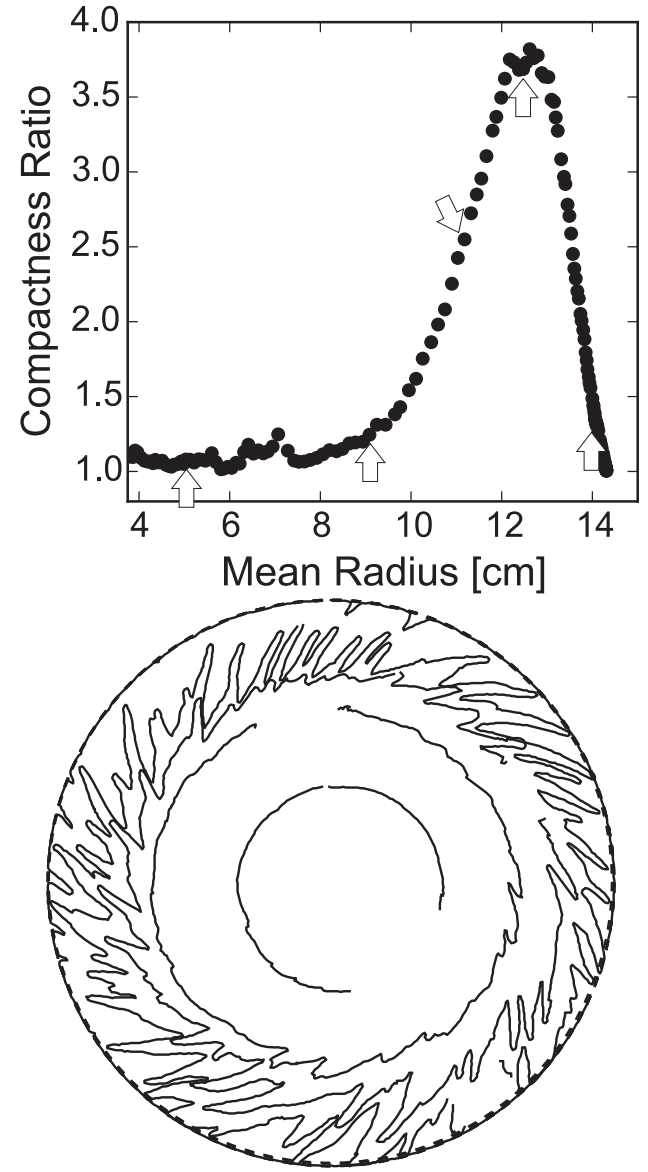

FIG. 6. A representative example of the compactness ratio vs mean radius. Contours of the spreading front traced by image-processing software are shown superimposed on a single image below, with their corresponding mean radii from inside to outside indicated sequentially by arrows along the bottom of the plot from left to right. Around each contour, the edge of the viewing window is demarcated by a dashed line (radius is $14 \mathrm{~cm}$ ). The substrate was bare, the flow rate of the impinging jet was $840 \mathrm{ml} / \mathrm{min}$, and the rotation rate was $250 \mathrm{rpm}$.

below. The compactness ratio therefore provides a simple metric of rivulet growth to make quantitative comparisons among different experiments.

\section{Evolution of the compactness ratio}

The evolution of the compactness ratio typically follows the behavior shown in Fig. 6. Varying system parameters like the surface condition, flow rate of the jet, and rotation rate of the substrate quantitatively affects the evolution of the compactness ratio. We demonstrate these effects by plotting the compactness ratio vs the mean radius for several representative examples in Fig. 7.

We first discuss the effect of the surface condition. To assist with this discussion, Fig. 7 shows contours of the spreading front on different surface conditions at the same flow rate and rotation rate. The corresponding mean radii are marked by the arrows at the bottom of the plot. From the contours, we see 


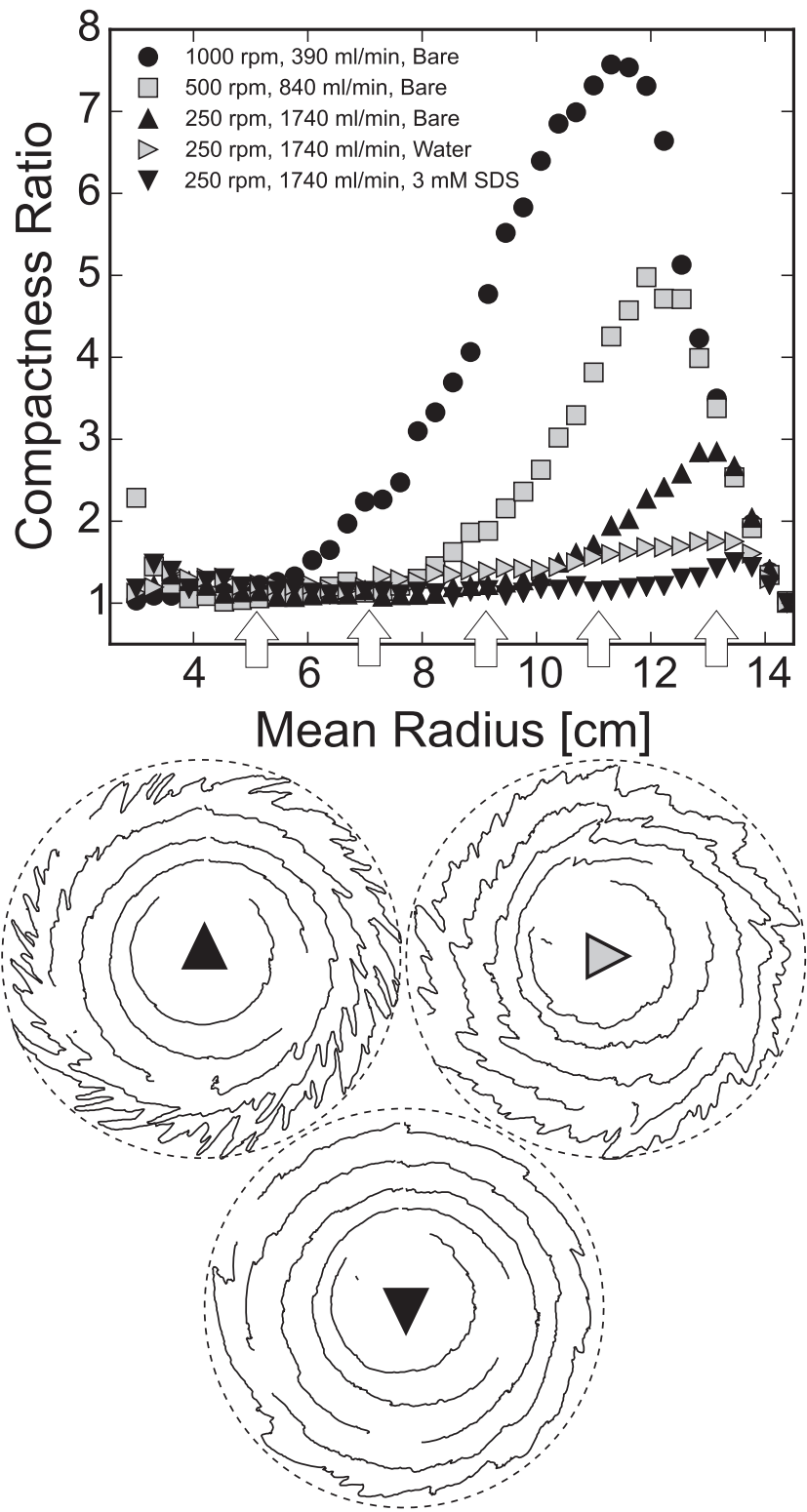

FIG. 7. Compactness ratio as a function of the mean radius of the spreading front for different surface conditions, flow rates, and rotation rates. Radii below $3 \mathrm{~cm}$ are not shown because the spreading front could not be fully identified by the imageprocessing software. Contours in the reference frame of the substrate are shown below for the three different surface conditions at the mean radii marked by the arrows at the bottom of the plot. The corresponding conditions are indicated by the symbols in the centers of the contours.

that the rivulets were longest on the bare substrate. Accordingly, the compactness ratio for the bare substrate reached the highest value around 2, as compared to 1.5 for the water-coated substrate and 1.3 for the SDS-coated substrate. Nevertheless, undulations along the spreading front were observed earliest on the watercoated substrate, as seen in the contours and indicated by the early increase in compactness ratio. The spreading front on the SDScoated substrate formed rivulets latest and its rivulets grew the least.

We offer the following explanations of these trends. We expect rivulets to form earliest on a film coated with water in this case because the spreading front is not stabilized by any interfacial tension. The interfacial tension along the spreading front when spreading over a film of SDS solution, however, stabilizes undulations, delaying the formation of rivulets. We expect rivulets to elongate most on a bare substrate because spreading over a solid surface is less favorable than spreading over a liquid film, so water will be most driven to flow down rivulets on a bare substrate. This reasoning suggests that the compactness ratio would generally be higher for SDS-coated substrates than for water-coated substrates due to the greater interfacial tension along an interface between water and SDS than between water and water. We only observe this trend at lower flow rates, however, as is discussed below in Fig. 9. In this case, where the flow rate is $1740 \mathrm{ml} / \mathrm{min}$ (turbulent), the compactness ratios for the two surface conditions are within the uncertainty, suggesting that resistance along the spreading front has less of an effect on the elongation of rivulets at higher flow rates.

Next, we show how the spreading front reaches the highest values of the compactness ratio when spreading on a bare substrate at high rotation rates and low flow rates. We demonstrate this progression in Fig. 7 with a series of experiments on a bare substrate in which the rotation rate was successively doubled and the flow rate was successively halved. The growth of the compactness ratio changed in three ways as a result of a higher rotation rate and lower flow rate. First, the critical radius decreased, consistent with the quantitative relationship presented by Togashi et al. ${ }^{22}$ Second, the rate of growth of the compactness ratio after the critical radius was reached increased. Third, the previous two concurrent effects resulted in a higher peak compactness ratio. While the compactness ratio varied quantitatively, the qualitative shape of its trajectory remained unchanged, supporting the idea that the mechanism of rivulet growth is similar for these three cases of spreading on a bare substrate.

\section{Effect of splash on rivulets}

Section III B 4 focused on the compactness ratio of spreading fronts with rivulets formed primarily by the centrifugal force. Nevertheless, we observed that rivulets can also form during the initial impact of the impinging jet on the substrate, particularly at higher flow rates. We call this type of rivulet formation "splashing."

To examine the effect of splashing on rivulet formation, we compare the evolution of the compactness ratio for experiments with and without splashing in Fig. 8. The figure shows the contours of the spreading front traced by image processing at the mean radii marked by the arrows at the bottom of the plot. We compare low and high flow rates as well as stationary and rotating substrates. At a low flow rate on a stationary substrate, the spreading front was mostly circular. At a high flow rate on a stationary substrate, the spreading front initially splashed outward after the impact of the impinging jet, but the splash was subsequently filled and the spreading front became circular. At a low flow rate on a rotating substrate, the spreading front was initially circular, but gradually rivulets 

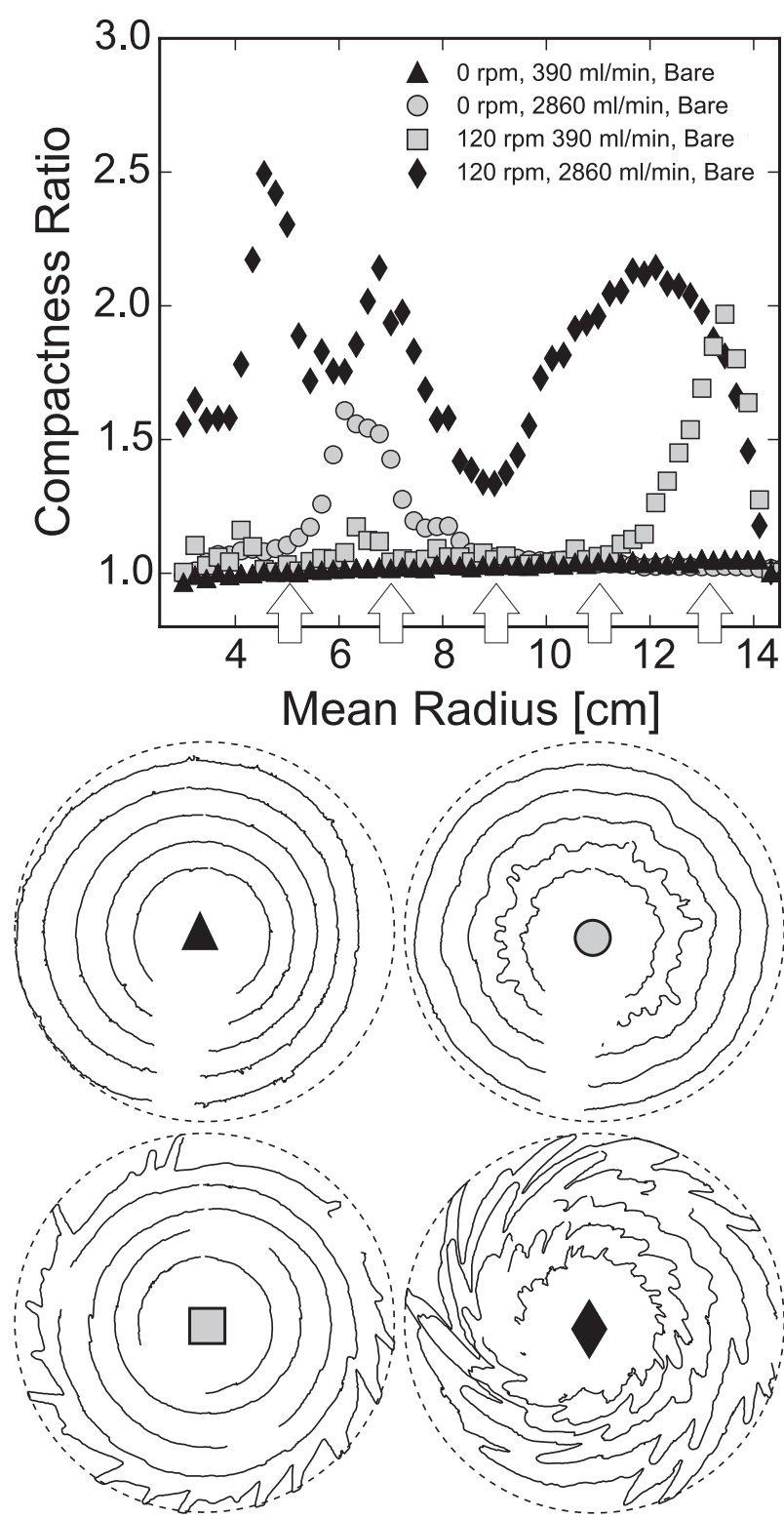

FIG. 8. The compactness ratio as a function of the mean radius of the spreading front on a bare substrate for: triangle $-0 \mathrm{rpm}, 390 \mathrm{ml} / \mathrm{min}$, no splash or rivulets; circle-0 rpm, $2860 \mathrm{ml} / \mathrm{min}$, initial splash is later filled in (note that off-center spreading is caused by a slight tilt of the substrate); square-120 rpm, $390 \mathrm{ml} / \mathrm{min}$, no splash, rivulets form later; diamond-120 rpm, $2860 \mathrm{ml} / \mathrm{min}$, initial splash is partially filled in before it is pulled into rivulets by the centrifugal force. Contours of the spreading front in the reference frame of the substrate are shown below for the mean radii marked with arrows $(5 \mathrm{~cm}, 7 \mathrm{~cm}, 9 \mathrm{~cm}, 11 \mathrm{~cm}$, and $13 \mathrm{~cm})$ at the bottom of the plot. The corresponding conditions are indicated by the symbols at the centers of the contours. Data for radii below about $3 \mathrm{~cm}$ are not shown because the image-processing software could not identify the full spreading front.

formed and elongated. At a high flow rate on a rotating substrate, the spreading front initially splashed outward and was subsequently filled in partially. Before the undulations of the initial splash were filled in, however, the centrifugal force pulled them outward into rivulets. The spreading front under this last condition resembled a combination of the spreading fronts under the second and third conditions.

We compare these qualitative observations of the spreading front to now plots of the compactness ratio vs time. For a stationary substrate and a low flow rate $(390 \mathrm{ml} / \mathrm{min})$, the compactness ratio remained roughly constant around one, matching the roughly circular contours. When the flow rate was increased to $2860 \mathrm{ml} / \mathrm{min}$, the compactness ratio increased after the initial impact, indicating the splash of the impinging jet. When spinning the substrate $(120 \mathrm{rpm})$ and having a low jet flow rate $(390 \mathrm{ml} / \mathrm{min})$, the compactness ratio did not increase until reaching about two-thirds of the viewing window, at which point the stronger centrifugal force caused rivulets to form. Finally, at a high flow rate $(2860 \mathrm{ml} / \mathrm{min})$ on a rotating substrate $(120 \mathrm{rpm})$, the compactness ratio peaks three times. The first two peaks occur at smaller radii where a splash is observed in the corresponding contours of the spreading front. The first occurs shortly after the initial impact of the impinging jet. The second is a result of a small pulse in the jet flow rate, which was sometimes observed upon start-up at the highest flow rate due to limitations of the pump. The detection of this small fluctuation in rivulet formation demonstrates the sensitivity of the measurement of the compactness ratio to changes in the hydrodynamics of the system. After these peaks, the compactness ratio decreased slightly before the last peak. Thus, the splash was only partially filled in before the centrifugal force pulled the remaining undulations into rivulets.

These observations show that splashing from the impinging jet and rotation of the substrate cause qualitatively similar increases in the compactness ratio, although at different radii. The smaller radii of peaks in the compactness ratio due to splashing than peaks due to the centrifugal force allows these two effects to be distinguished. Nevertheless, in some cases, rivulets that formed due to splashing were not filled in completely before the centrifugal force elongated them, so the initial splash caused the compactness ratio to increase overall. For this reason, studying how the flow rate can be varied over time to control the splash from the initial impact of the impinging jet will be important for future work in this field.

\section{Comparing the effects of rotation rate, flow rate, and surface condition}

To compare the effects of the rotation rate, flow rate, and surface condition on the compactness ratio, we focus on the value of the compactness ratio at a single point in time, that described in Sec. III A 2, which we call the "peak compactness ratio."

In Fig. 9, we plot the peak compactness ratio as a function of the flow rate. We separate the three surface conditions-bare, coated with $3 \mathrm{mM}$ SDS, and coated with water-into three plots for clarity. Different rotation rates are represented by different symbols. For bare substrates and SDS-coated substrates, the peak compactness ratio followed similar qualitative trends, although the values were lower for SDS-coated substrates. For both surface conditions, the compactness ratio increased with the rotation rate, most markedly at low flow rates, although the difference in compactness ratio at $500 \mathrm{rpm}$ and $1000 \mathrm{rpm}$ is below the uncertainty. Additionally, increasing the flow rate decreased the compactness ratio for rotation rates of $500 \mathrm{rpm}$ and above, while it had no noticeable effect on the compactness ratio for lower rotation rates. 


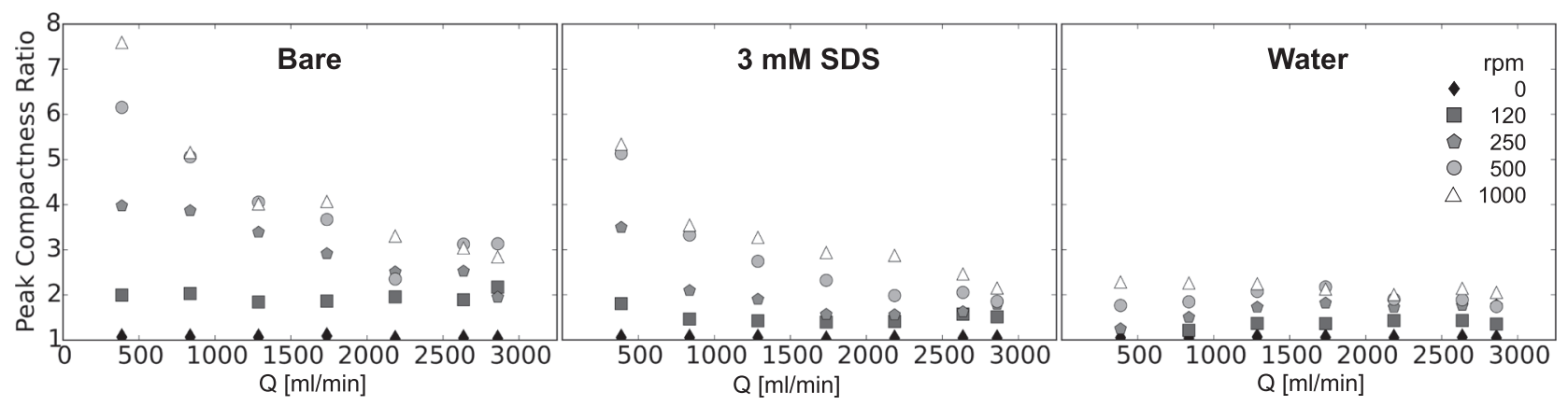

FIG. 9. Peak compactness ratio as a function of the flow rate of the impinging jet $(\mathrm{m} / / \mathrm{min})$ for different surface conditions and rotation rates. Left bare substrate. Center substrate coated with $3 \mathrm{mM}$ SDS aqueous solution. Right substrate coated with water. The symbol and its shade indicate the rotation rate given in the legend on the right.

In the case of substrates coated with water, the compactness ratio was generally smaller than for bare or SDS-coated substrates, although in some experiments, the compactness ratio was smaller on SDS-coated substrates for the same flow rate and rotation rate (see Fig. 7). The compactness ratio also generally increased with rotation rate, although not as markedly as for the other two surface conditions. Contrary to the other two surface conditions, the flow rate of the jet did not have a noticeable effect on the compactness ratio for any rotation rate.

We suggest the following explanations for the trends observed. First, a higher rotation rate increases the compactness ratio because the centrifugal force increases at higher rotation rates. As centrifugal force increases, rivulets form earlier, as observed in both this study and previous studies. ${ }^{12,13,22}$ As a result, rivulets begin to grow earlier as well. Additionally, the increase in the gradient of the centrifugal force with radius causes rivulets to extend beyond the lagging edge of the spreading front more quickly at higher rotation rates.

Second, we suggest that the higher hydrodynamic pressure from the flow of the jet at higher flow rates is responsible for the decrease in compactness ratio with flow rate at rotation rates above $120 \mathrm{rpm}$ on bare and SDS-coated substrates. Because hydrodynamic pressure is strongest near the jet, we expect that it pushes the lagging edge of the spreading front more strongly than the leading edge of the rivulets, shortening rivulets and decreasing the compactness ratio, consistent with our observations. We do not observe any noticeable effect of the flow rate on the compactness ratio at rotation rates of $120 \mathrm{rpm}$ and below. We suggest that, due to the weaker centrifugal force, the splash from the initial impact of the impinging jet on the substrate (discussed further below) plays a relatively more pronounced role in forming rivulets. Therefore, at lower rotation rates, the suppression of rivulets at higher flow rates may be counteracted by the concomitant increase in the splash from the initial impact of the jet on the substrate, resulting in no noticeable overall effect on the peak compactness ratio.

Third, we suggest that the reason for the general decrease in compactness ratio in the order of bare, SDS-coated, water-coated for nonturbulent impinging jets (flow rate of $1000 \mathrm{ml} / \mathrm{min}$ or below) is the decreasing resistance to flow along the spreading front. This general trend is consistent with previous observations that spreading over a thin film of a similar liquid suppresses the instability that leads to rivulets, as compared to spreading over a bare, solid substrate. ${ }^{12,19,26}$ For these flow rates, the capillary ridge appeared to be less pronounced the lower the resistance along the spreading front, as expected. Because rivulets form due to fluctuations in the height of the capillary ridge, ${ }^{5}$ we would expect rivulets to be suppressed when the capillary ridge is thinner, as observed for water-coated substrates. This hypothesis is also consistent with the results of Arora and Doshi, who showed numerically that during the spin coating of power-law fluids, the formation of rivulets was suppressed for the thinner capillary ridges observed with fluids having lower power laws (shear-thinning). ${ }^{37}$ Additionally, we would expect that coating the substrate with an aqueous liquid film reduces the preference of the flow to funnel down rivulets, which may explain why we generally observed higher compactness ratios for a bare substrate than an SDS-coated substrate.

Finally, at turbulent flow rates (above $1000 \mathrm{ml} / \mathrm{min}$ ), the compactness ratio is similar for both SDS-coated and water-coated substrates. This observation seemingly contradicts our discussion above, which suggested that rivulets do not form as readily or elongate as quickly on water-coated substrates as they do on SDScoated surfaces. We believe this discrepancy is the result of a larger splash from the initial impact of the impinging jet on watercoated substrates than on SDS-coated substrates. This explanation is supported both by visual observation and by the earlier increase in the compactness ratio for water-coated substrates, an example of which can be seen in Fig. 7. A larger splash would increase the formation of rivulets on water-coated substrates, which could balance the effects that increase rivulet formation and elongation on SDS-coated substrates that we discussed above. With a larger substrate, this explanation could be validated by showing that at larger radii, the compactness ratio is higher for SDS-coated substrates.

\section{Morphology of the spreading front and the compactness ratio}

Finally, we consider how the compactness ratio varies for different morphologies of the spreading front. Figure 10 shows a jitter plot of the peak compactness ratio for spreading fronts classified into the seven categories proposed above. This comparison shows us that the compactness ratio increases roughly in the same order of our qualitative categorization of the aspect ratio of rivulets from 


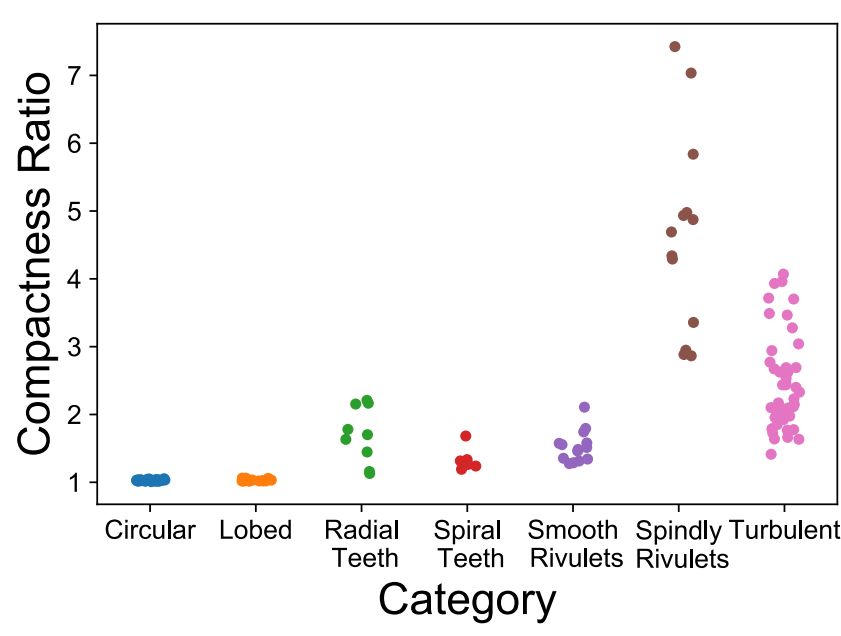

FIG. 10. Jitter plot of the compactness ratio for each category. The horizontal distribution of data points within a category is randomly assigned to permit the reader to distinguish data points with similar values. One data point was obtained for each unique set of system parameters, excluding cases for which the morphology was determined to be in "transition" in Fig. 4.

categories 1-6. We also see that despite the larger spread in the compactness ratios of spindly rivulets, they are noticeably higher than those of the other categories. Circular and lobed spreading fronts both have clearly lower compactness ratios than the other categories. Nevertheless, Fig. 10 shows that several categories of spreading front morphology have the same compactness ratio.

In particular, the compactness ratios of "radial teeth," "spiral teeth," "smooth rivulets," and "turbulent rivulets" overlap from 1.2 to 1.8. From the examples in Fig. 4, we see that the spreading fronts from these categories can have drastically varying morphologies, such as many short radial rivulets, few long spiralled rivulets, and variations in between. The matching values of the compactness ratio for vastly different morphologies can be understood by visualizing a length of string connected between two points in a plane separated by a distance smaller than the length of the string. There are infinitely many configurations of the string that enclose the same area on either side of the string. In these cases, the area enclosed and the perimeter are the same, as in the case of spreading fronts with equal compactness ratios. It is therefore not surprising that using only a single parameter does not provide enough degrees of freedom to precisely quantify the morphology of the entire spreading front. This observation underscores the importance of also qualitatively analyzing the morphology of the spreading front to capture the richness of this phenomenon. Nevertheless, we believe that the compactness ratio provides a useful quantitative measurement for evaluating and tracking rivulets.

\section{CONCLUSION}

In conclusion, we have elucidated several key features of the spreading of liquid supplied by an impinging jet on a rotating substrate. In particular, we have evaluated the effects of the flow rate of the impinging jet, the rotation rate of the substrate, and precoating the substrate with aqueous films.
Qualitatively, we classified the morphology of the spreading fronts we observed into seven distinct categories based on the aspect ratio, degree of spiraling, and regularity of the rivulets that were formed. We found that many features of these morphologies could be explained by the conditions of spreading, with particular attention to the Rossby number and Reynolds number.

To analyze the morphology of the spreading front quantitatively, we developed an apparatus and software that could precisely distinguish the spreading front of water from the aqueous liquid films precoated on the substrate. This capability allowed us to measure the "compactness ratio" of the spreading front, which we have studied in depth for the first time in this field. We showed that the compactness ratio strongly correlates with the formation and elongation of rivulets, which motivated our use of the compactness ratio to quantify the effect of different system parameters on these processes. Through this analysis, we showed that, in general, the compactness ratio is lower for spreading over a substrate that is precoated with a thin film of the rinsing liquid (water), highlighting the importance of contact line forces and their effect on the capillary ridge that precedes rivulet formation.

Additionally, we showed that the compactness ratio generally increases with the rotation rate of the substrate-albeit to a lesser degree at higher rotation rates-highlighting the central role played by centrifugal force in forming and elongating rivulets.

Finally, we found that while increasing the flow rate decreases the compactness ratio at high rotation rates on bare and SDS-coated substrates, at lower flow rates and on water-coated substrates, the effect of flow rate on compactness ratio is negligible. In these cases, the effect is likely negligible because of the competition between the opposing effects of greater suppression of rivulet elongation and larger initial splash on the compactness ratio at higher flow rates. Based on our observation that the spreading front transitions through several distinct morphologies during spreading, however, we would expect these trends to vary for spreading at radii larger than the substrate considered here.

While providing some speculative explanations of the phenomena observed, the present work primarily seeks to describe the rich diversity of morphologies adopted by the rivulets that form in this system, as well as the effects of system parameters thereon. Nevertheless, rigorous explanation of both previously and newly reported phenomena presented in this paper will require deeper theoretical analysis of this system; we hope that this work will motivate such studies.

\section{ACKNOWLEDGMENTS}

The authors are grateful to the Lam Research Corporation for support in the form of an unrestricted gift. The authors would also like to thank Brian Friedenberg for assistance with the image-processing software. This work was partially funded by the National Science Foundation CBET Grant Nos. 1335632 and 1435683.

\section{REFERENCES}

${ }^{1}$ R. V. Craster and O. K. Matar, "Dynamics and stability of thin liquid films," Rev. Mod. Phys. 81, 1131-1198 (2009).

${ }^{2}$ A. Oron, S. H. Davis, and S. G. Bankoff, "Long-scale evolution of thin liquid films," Rev. Mod. Phys. 69, 931-980 (1997). 
${ }^{3}$ A.-M. Cazabat, F. Heslot, S. M. Troian, and P. Carles, "Fingering instability of thin spreading films driven by temperature gradients," Nature 346, 824-826 (1990).

${ }^{4}$ A. A. Darhuber and S. M. Troian, "Marangoni driven structures in thin film flows," Phys. Fluids 15, 1295 (2003).

${ }^{5}$ S. M. Troian, E. Herbolzheimer, S. A. Safran, and J.-F. Joanny, "Fingering instabilities of driven spreading films," Europhys. Lett. 10, 25-30 (1989).

${ }^{6}$ J. A. Diez, A. G. González, and L. Kondic, "On the breakup of fluid rivulets," Phys. Fluids 21, 82105 (2009).

${ }^{7}$ H. E. Huppert, "Flow and instability of a viscous current down a slope," Nature 300, 427-429 (1982).

${ }^{8} \mathrm{~N}$. Silvi and E. B. Dussan, "The rewetting of an inclined solid surface by a liquid," Phys. Fluids 28, 5 (1985).

${ }^{9}$ L. M. Hocking, W. R. Debler, and K. E. Cook, "The growth of leading-edge distortions on a viscous sheet," Phys. Fluids 11, 307 (1999).

${ }^{10} \mathrm{~S}$. K. Wilson and B. R. Duffy, "Unidirectional flow of a thin rivulet on a vertical substrate subject to a prescribed uniform shear stress at its free surface," Phys. Fluids 17, 108105 (2005).

${ }^{11}$ D. Takagi and H. E. Huppert, "Flow and instability of thin films on a cylinder and sphere," J. Fluid Mech. 647, 221 (2010).

${ }^{12}$ F. Melo, J.-F. Joanny, and S. Fauve, "Fingering instability of spinning drops," Phys. Rev. Lett. 63, 1958-1961 (1989).

${ }^{13}$ N. Fraysse and G. M. Homsy, "An experimental study of rivulet instabilities in centrifugal spin coating of viscous Newtonian and non-Newtonian fluids," Phys. Fluids 6, 1491-1504 (1994).

${ }^{14}$ M. A. Spaid and G. M. Homsy, "Stability of viscoelastic dynamic contact lines: An experimental study," Phys. Fluids 9, 823 (1998).

${ }^{15}$ D. S. L. Mui, J. Zhu, and A. Mendiratta, "Materials and systems for advanced substrate cleaning," U.S. patent 8314055B2 (November 20, 2012).

${ }^{16}$ M. Mirzadeh and M. Z. Bazant, "Electrokinetic control of viscous fingering," Phys. Rev. Lett. 119, 174501 (2017).

${ }^{17}$ D. P. Jackson and J. A. Miranda, "Controlling fingering instabilities in rotating ferrofluids," Phys. Rev. E 67, 017301 (2003).

${ }^{18}$ K. E. Holloway, P. Habdas, N. Semsarillar, K. Burfitt, and J. R. de Bruyn, "Spreading and fingering in spin coating," Phys. Rev. E 75, 046308 (2007).

${ }^{19}$ R. H. de Bruijne and J. H. Lammers, "Experiments on finger formation during spin coating," Technical Report No. 807/99, Philips Electronics, 1999.

${ }^{20}$ H.-C. Cho, F.-C. Chou, M.-W. Wang, and C.-S. Tsai, "Effect of Coriolis force on fingering instability and liquid usage reduction," Jpn. J. Appl. Phys., Part 2 44, L606-L609 (2005).

${ }^{21}$ H.-C. Cho and F.-C. Chou, "Rivulet instability with effect of Coriolis force," J. Mech. 22, 221-227 (2006).
${ }^{22}$ S. Togashi, T. Ohta, and H. Azuma, "Fingering flow pattern of a liquid film on a rotating substrate," J. Chem. Eng. Jpn. 34, 1402-1406 (2001).

${ }^{23}$ F.-C. Chou, M.-W. Wang, S.-C. Gong, and Z.-G. Yang, "Reduction of photoresist usage during spin coating," J. Electron. Mater. 30, 432-438 (2001).

${ }^{24}$ M.-W. Wang, J.-L. Ma, and L.-W. Hourng, "Effects of dispensing flow rate on fingering instability during spin coating," Jpn. J. Appl. Phys., Part 1 53, 036501 (2014).

${ }^{25} \mathrm{D}$. E. Weidner, "Analysis of the flow of a thin liquid film on the surface of a rotating, curved, axisymmetric substrate," Phys. Fluids 30, 082110 (2018).

${ }^{26}$ I. Veretennikov, A. Agarwal, A. Indeikina, and H.-C. Chang, "Unusual contactline dynamics of thick films and drops," J. Colloid Interface Sci. 215, 425-440 (1999).

${ }^{27}$ S. Sahoo, A. V. Orpe, and P. Doshi, "Spreading dynamics of superposed liquid drops on a spinning disk," Phys. Fluids 30, 012110 (2018).

${ }^{28}$ S. Sahoo, A. Arora, and P. Doshi, "Two-layer spin coating flow of Newtonian liquids: A computational study," Comput. Fluids 131, 180-189 (2016).

${ }^{29}$ D. J. Walls, A. S. Ylitalo, D. S. L. Mui, J. M. Frostad, and G. G. Fuller, "Spreading of rinsing liquids across a horizontal rotating substrate," Phys. Rev. Fluids $\mathbf{4}$, 084102 (2019).

${ }^{30}$ T. T. Hsu, T. W. Walker, C. W. Frank, and G. G. Fuller, "Role of fluid elasticity on the dynamics of rinsing flow by an impinging jet," Phys. Fluids 23, 033101 (2011).

${ }^{31}$ T. W. Walker, T. T. Hsu, C. W. Frank, and G. G. Fuller, "Role of shear-thinning on the dynamics of rinsing flow by an impinging jet," Phys. Fluids 24, 093102 (2012).

${ }^{32}$ J. M. Frostad, D. Tammaro, L. Santollani, S. Bochner de Araujo, and G. G. Fuller, "Dynamic fluid-film interferometry as a predictor of bulk foam properties," Soft Matter 12, 9266-9279 (2016).

${ }^{33}$ A. G. Emslie, F. T. Bonner, and L. G. Peck, "Flow of a viscous liquid on a rotating disk," J. Appl. Phys. 29, 858-862 (1958).

${ }^{34}$ M. Kulkarni, S. Sahoo, P. Doshi, and A. V. Orpe, "Fingering instability of a suspension film spreading on a spinning disk," Phys. Fluids 28, 063303 (2016).

${ }^{35} \mathrm{C}$. Dai, Y. Zheng, and X. Li, "Pedestrian detection and tracking in infrared imagery using shape and appearance," Comput. Vision Image Understanding 106, 288-299 (2007).

${ }^{36} \mathrm{H}$. Possingham, I. Ball, and S. Andelman, "Mathematical methods for identifying representative reserve networks," in Quantitative Methods for Conservation Biology, edited by S. Ferson and M. Burgman (Springer-Verlag, New York, 2000), Chap. 17, pp. 291-306.

${ }^{37}$ A. Arora and P. Doshi, "Fingering instability in the flow of a power-law fluid on a rotating disc," Phys. Fluids 28, 013102 (2016). 